\title{
SUPERCRITICAL HOLES FOR THE DOUBLING MAP
}

\author{
NIKITA SIDOROV
}

\begin{abstract}
For a map $S: X \rightarrow X$ and an open connected set (= a hole) $H \subset X$ we define $\mathcal{J}_{H}(S)$ to be the set of points in $X$ whose $S$-orbit avoids $H$. We say that a hole $H_{0}$ is supercritical if

(i) for any hole $H$ such that $\overline{H_{0}} \subset H$ the set $\mathcal{J}_{H}(S)$ is either empty or contains only fixed points of $S$;

(ii) for any hole $H$ such that $\bar{H} \subset H_{0}$ the Hausdorff dimension of $\mathcal{J}_{H}(S)$ is positive.

The purpose of this note is to completely characterize all supercritical holes for the doubling map $T x=2 x \bmod 1$.
\end{abstract}

\section{INTRODUCTION AND INITIAL RESULTS}

The present paper is concerned with an area of dynamics which is usually referred to as "open dynamical systems" or, more colloquially, "maps with holes". Let us remind the reader the basic set-up: let $X$ be a compact (or precompact) metric space and $S: X \rightarrow X$ be a map with positive topological entropy (for the definition of topological entropy see, e.g., [6, Definition 3.1.3]). Let $H \subset X$ be an open connected set which we treat as a hole.

We denote by $\mathcal{J}_{H}(S)$ the set of all points in $X$ whose $S$-orbit does not intersect $H$. In other words,

$$
\mathcal{J}_{H}(S)=X \backslash \bigcup_{n=-\infty}^{\infty} S^{-n} H
$$

if $S$ is invertible or

$$
\mathcal{J}_{H}(S)=X \backslash \bigcup_{n=0}^{\infty} S^{-n} H
$$

Date: November 20, 2018.

2010 Mathematics Subject Classification. Primary 28D05; Secondary 37B10, $68 \mathrm{R} 15$.

Key words and phrases. Open dynamical system, doubling map, Sturmian system, characteristic word, supercritical hole. 
if it is not. Clearly, $\mathcal{J}_{H}(S)$ is $S$-invariant, and in a number of recent papers certain dynamical properties of the exclusion map $\left.S\right|_{\mathcal{J}_{H}(S)}$ have been studied - see, e.g., [3] and the references therein.

We believe a more immediate issue here is the "size" of the set $\mathcal{J}_{H}(S)$ - after all, if it is countable (or, even worse, empty), any questions regarding the dynamics of the exclusion map become uninteresting.

Let $X=[0,1)$ and $T x=2 x \bmod 1$, the famous doubling map. Assume first that $H$ is a symmetrical interval about $1 / 2$, i.e., $H=H_{a}:=$ $(a, 1-a)$ for some $a \in(0,1 / 2)$. Note first that if $a<1 / 3$, then $\mathcal{J}_{H_{a}}(T)=\{0\}$, since for any $x$ in $\left(a / 2^{n}, a / 2^{n-1}\right)$ or in $\left(1-a / 2^{n-1}, 1-\right.$ $\left.a / 2^{n}\right)$ with $n \geq 1$, we have $T^{n}(x) \in(a, 2 a)$ or $(1-2 a, 1-a)$, both being subsets of $H_{a}$.

On the other hand, if $a \geq 1 / 3$, then the 2-cycle $\{1 / 3,2 / 3\}$ does not intersect $H_{a}$. Let us give a general definition:

Definition 1.1. Let $\operatorname{Fix}(S)=\{x \in S: S x=x\}$. We say that a hole $H_{0}$ is first order critical for $S$ if

(i) for any hole $H$ such that $\overline{H_{0}} \subset H$ we have $\mathcal{J}_{H}(S) \subset \operatorname{Fix}(S)$;

(ii) for any hole $H$ such that $\bar{H} \subset H_{0}$ we have $\mathcal{J}_{H}(S) \not \subset \operatorname{Fix}(S)$.

Thus, $H_{1 / 3}$ is a first order critical hole for $T$. Note that if $a \in$ $(1 / 3,2 / 5)$, then $\mathcal{J}_{H_{a}}(T)=\mathcal{J}_{H_{1 / 3}}(T)$, i.e., just the 2 -cycle and all its preimages - see [1]. If we keep increasing a towards $1 / 2$, we start getting more and more cycles in $\mathcal{J}_{H_{a}}(T)$.

Let us introduce some symbolic dynamics. Namely, put $\Sigma=\{0,1\}^{\mathbb{N}}$ and let $\sigma$ denote the one-sided shift on $\Sigma$, i.e., $\sigma\left(w_{1}, w_{2}, w_{3}, \ldots\right):=$ $\left(w_{2}, w_{3}, \ldots\right)$. Let $\pi: \Sigma \rightarrow[0,1]$ be defined as follows:

$$
\pi\left(w_{1}, w_{2}, \ldots\right)=\sum_{k=1}^{\infty} w_{k} 2^{-k}
$$

Then, as is well known, $\pi$ in one-to-one on all sequences except a countable set, and $\pi \sigma=T \pi$. Thus, there is one-to-one correspondence between the cycles for $T$ and those for $\sigma$. If $a \geq 2 / 5$, then the $\pi$-image of the 4-cycle 0110 for $\sigma$ lies outside the hole. The next one to appear is 01101001 . etc.

Once we have run through all these $2^{n}$-cycles, we reach another critical value, $a_{*} \approx 0.412454$ whose binary expansion is the famous ThueMorse sequence:

$011010011001011010010110 \ldots$ 
It is known that if $a<a_{*}$, then $\mathcal{J}_{H_{a}}(T)$ is infinite countable; if $a>$ $a_{*}$, then $\operatorname{dim}_{H}\left(\mathcal{J}_{H_{a}}(T)\right)>0-$ see [12, 10, 4] $]$. This calls for another definition:

Definition 1.2. We say that a hole $H_{0}$ is second order critical for $S$ if

(i) for any hole $H$ such that $\overline{H_{0}} \subset H$ we have $\operatorname{dim}_{H}\left(\mathcal{J}_{H}(S)\right)=0$;

(ii) for any hole $H$ such that $\bar{H} \subset H_{0}$ we have $\operatorname{dim}_{H}\left(\mathcal{J}_{H}(S)\right)>0$.

Remark 1.3. Definitions 1.1 and 1.2 are in the same spirit as those of functions $\varphi$ and $\chi$ in [7].

Consequently, $\left(a_{*}, 1-a_{*}\right)$ is a second order critical hole for $T$. We see that there is a substantial distance between the first order and second order symmetrical critical holes for the doubling map. If we regard $a$ as time, then one may say that it takes long for $T$ to accommodate all these cycles, quite in line with the standard notion of "route to chaos via period doubling" common in one-dimensional dynamics. For more details see [1].

Let us give the central definition of this note which in a way combines the previous ones:

Definition 1.4. We say that a hole $H_{0}$ is supercritical for $S$ if

(i) for any hole $H$ such that $\overline{H_{0}} \subset H$ we have $\mathcal{J}_{H}(S) \subset \operatorname{Fix}(S)$;

(ii) for any hole $H$ such that $\bar{H} \subset H_{0}$ we have $\operatorname{dim}_{H}\left(\mathcal{J}_{H}(S)\right)>0$.

In other words, a supercritical hole is a hole which is both of first and second order. Since from here on we only consider the doubling map, we would like to simplify our notation and write $\mathcal{J}(H)$ instead of $\mathcal{J}_{H}(T)$ or simply $\mathcal{J}(a, b)$ if $H=(a, b)$. Our first result yields a simple family of supercritical holes:

Proposition 1.5. For each $a \in[0,1 / 4]$, the holes $H_{0}=(a, 1 / 2)$ and $H_{0}^{\prime}=(1 / 2,1-a)$ are supercritical for the doubling map $T$.

Proof. We will prove the first claim; the second one is obtained by swapping 0 and 1. Let us check both conditions of Definition 1.4.

(i) Suppose $\overline{H_{0}} \subset H$; then $[1 / 4,1 / 2] \subset H$, which means that each point $x \in H$ whose $T$-orbit avoids $H$ cannot have 01 in its binary expansion. Hence $x=0$, because if the binary expansion of $x$ ends with $10^{\infty}$, it can be replaced with $01^{\infty}$, which lies in $H$.

(ii) Let $\bar{H} \subset H_{0}$. Fix $n \geq 2$ and consider the following subshift of finite type:

$$
\Sigma_{n}=\left\{w \in \Sigma: w_{k}=0 \Longrightarrow w_{k+j}=1, j=1, \ldots, n\right\} .
$$

\footnotetext{
${ }^{1}$ Technically, 4] deals with the space of unique $\beta$-expansions but the symbolic model is essentially the same.
} 
When each 0 in the binary expansion of some $x \in(0,1)$ is succeeded by at least $n$ consecutive $1 \mathrm{~s}$, this means that $x \geq \frac{2^{-2}+2^{-3}+\cdots+2^{-n}}{1-2^{-n-1}}$. Thus,

$$
\pi\left(\Sigma_{n}\right) \subset\left[\frac{\frac{1}{2}-2^{-n-1}}{1-2^{-n-1}}, 1\right)
$$

which implies that $\pi\left(\Sigma_{n}\right) \subset \mathcal{J}(H)$ for all $n$ large enough. The topological entropy $h_{\text {top }}$ of the subshift $\left.\sigma\right|_{\Sigma_{n}}$ is positive, whence $\operatorname{dim}_{H} \mathcal{J}(H) \geq$ $\operatorname{dim}_{H} \pi\left(\Sigma_{n}\right)=h_{\text {top }}\left(\left.\sigma\right|_{\Sigma_{n}}\right) / \log 2>0$.

In the next section we will construct a continuum of supercritical holes for $T$ parametrized by Sturmian sequences. In Section 3 we present a complete list of supercritical holes for $T$.

\section{Sturmian HOLES}

For our purposes we need to define Sturmian systems. We will adapt our exposition to our needs; for a detailed survey and proofs of the auxiliary results stated below see [8, Chapter 2].

We say that a finite word $u$ is a factor of $w$ if there exists $k$ such that $u=w_{k} \ldots w_{k+n}$ for some $n \geq 0$. For a finite word $w$ let $|w|$ denote the length of $w$ and let $|w|_{1}$ stand for the number of 1 s in $w$. A finite or infinite word $w$ is balanced if for any $n \geq 1$ and any two factors $u, v$ of $w$ of length $n$ we have $\left.|| u\right|_{1}-|v|_{1} \mid \leq 1$.

Let $\gamma \in(0,1 / 2) \backslash \mathbb{Q}$ and let its continued fraction expansion be denoted by $\left[d_{1}+1, d_{2}, d_{3}, \ldots\right]$ with $d_{1} \geq 1$ (in view of $\gamma<1 / 2$ ). Let $p_{n} / q_{n}$ stand for the finite continued fraction $\left[d_{1}+1, \ldots, d_{n}\right]$ (in least terms). We define the sequence of $0-1$ words given by $\gamma$ as follows: $s_{-1}=1, s_{0}=0, s_{n+1}=s_{n}^{d_{n+1}} s_{n-1}, n \geq 0$. The word $s_{n}$ is called the $n$th standard word given by $\gamma$.

It is well known that $\left|s_{n}\right|_{1}=p_{n}$ and $\left|s_{n}\right|=q_{n}$ for every $n \geq 1$. Since $s_{n+1}$ begins with $s_{n}$ and $q_{n} \rightarrow \infty$, we conclude that there exists the limit $s_{\infty}(\gamma)=\lim _{n \rightarrow \infty} s_{n}$. This word is called the characteristic word given by $\gamma$. Note that any factor of a characteristic word is balanced.

Define the Sturmian system given by $\gamma$ as follows:

$$
X_{\gamma}=\overline{\left\{\sigma^{n} s_{\infty}(\gamma): n \in \mathbb{N}\right\}} .
$$

It is known that $X_{\gamma}$ is a perfect set and $\left.\sigma\right|_{X_{\gamma}}$ is minimal and has zero entropy. It is also known that any sequence $w$ in $X_{\gamma}$ which starts with 1 is a combination of blocks $10^{d_{1}}$ and $10^{d_{1}+1}$. If $w$ starts with 0 , it actually starts with $0^{d_{1}} 1$ or $0^{d_{1}+1} 1$, with the above rule valid for the rest of $w$. In particular, the blocks of 0's are bounded for a given $\gamma$.

It is obvious that $\left.\pi\right|_{X_{\gamma}}$ is an injection. From the above it follows that $K_{\gamma}:=\pi\left(X_{\gamma}\right)$ is a $T$-invariant Cantor set of zero Hausdorff dimension in 
$(0,1)$ which does not contain $1 / 2$ (since $1 / 2$ has the binary expansion with an unbounded number of 0's or 1's).

Remark 2.1. The set $K_{\gamma}$ has been considered in [2] where the authors proved in particular that any ordered $T$-invariant subset of $[0,1]$ must be $K_{\gamma}$ for some $\gamma$ or its finite version for $\gamma \in \mathbb{Q}$.

Recall that for $w, w^{\prime} \in \Sigma$ we have that $w$ is lexicographically less than $w^{\prime}$ if $w_{k}<w_{k}^{\prime}$ for the smallest $k$ such that $w_{k} \neq w_{k}^{\prime}$. (Notation: $w \prec w^{\prime}$.) It is clear that $w \preceq w^{\prime} \Longleftrightarrow \pi(w) \leq \pi\left(w^{\prime}\right)$.

Let $H_{0}(\gamma)$ denote the hole in $K_{\gamma}$ which contains $1 / 2$ (it is also the largest hole in $K_{\gamma}$ ). More precisely, let $0-\max (\gamma)$ denote the lexicographically largest sequence in $X_{\gamma}$ which starts with 0 ; similarly, we define $1-\min (\gamma)$. It is known (see [8, Chapter 2]) that the minimum sequence in $X_{\gamma}$ is in fact $0 s_{\infty}(\gamma)$ and the maximum is $1 s_{\infty}$. Consequently, $0-\max (\gamma)=01 s_{\infty}(\gamma)$ and $1-\min (\gamma)=10 s_{\infty}(\gamma)$, and $H_{0}(\gamma)=\left(\pi\left(01 s_{\infty}(\gamma)\right), \pi\left(10 s_{\infty}(\gamma)\right)\right)$. Clearly, the length of $H_{0}(\gamma)$ equals $1 / 4$.

Definition 2.2. We call $H_{0}(\gamma)$ the Sturmian hole associated with $\gamma$.

Example 2.3. Let $\gamma=(3-\sqrt{5}) / 2=[2,1,1,1, \ldots]$. Here $s_{n+1}=$ $s_{n} s_{n-1}$, which implies that $s_{\infty}(\gamma)$ is none other than the Fibonacci word

$$
f=010010100100101001010 \ldots
$$

Hence

$$
\begin{aligned}
\pi^{-1} H_{0}((3-\sqrt{5}) / 2)= & (01010010100100101001010 \ldots, \\
& 10010010100100101001010 \ldots),
\end{aligned}
$$

and $H_{0}\left(\frac{3-\sqrt{5}}{2}\right) \approx(0.322549,0.572549)$.

Definition 2.4. We say that a closed interval $I$ is a trap if $\bigcup_{k \geq 0} T^{-k}(I)=$ $(0,1)$.

Lemma 2.5. Let $\gamma=\left[d_{1}+1, d_{2}, \ldots\right]$. Fix $n \geq 2$ and put $r=\left[d_{1}+\right.$ $\left.1, \ldots, d_{n}\right]$. Set $s=s(r)=01 s_{n}, t=t(r)=10 s_{n}$. Then $\left[\pi\left(s^{\infty}\right), \pi\left(t^{\infty}\right)\right]$ is a trap.

Proof. Let $r_{1}<r<r_{2}$ be the Farey parents for $r$ and let $s_{i}=s\left(r_{i}\right), t_{i}=$ $t\left(r_{i}\right), i=1,2$. It is shown in [5, Lemma 3.2] that

$$
\begin{aligned}
& s=s_{1} t_{2}, \\
& t=t_{2} s_{1} .
\end{aligned}
$$


To prove the claim, we will use induction of the length of $s$. If $|s|=2$, then we have $r=\frac{1}{2}$, whence $\left[\pi\left(s^{\infty}\right), \pi\left(t^{\infty}\right)\right]=\left[\frac{1}{3}, \frac{2}{3}\right]$, about which we know that it is a trap - see above.

Assume now that the claim holds for all the rationals $r^{\prime}$ for which $\left|s\left(r^{\prime}\right)\right|<s(r)$. Consequently, it holds for $s_{1}$. Without loss of generality we may assume $x \in\left[\pi\left(s_{1}^{\infty}\right), \pi\left(t_{1}^{\infty}\right)\right]$. If $x \in\left[\pi\left(s^{\infty}\right), \pi\left(t^{\infty}\right)\right]$, we are done; otherwise note that $s_{1}^{\infty} \prec s^{\infty}$ and $t_{1}^{\infty} \prec t^{\infty}$, which is a well known property of standard words - see [8, Chapter 2]. Hence $x$ must lie in $\left[\pi\left(s_{1}^{\infty}\right), \pi\left(s^{\infty}\right)\right)$. Put $y=T^{\left|s_{1}\right|} x \in\left[\pi\left(s_{1}^{\infty}\right), \pi\left(t_{1}^{\infty}\right)\right)$. Again, if $y \in\left[\pi\left(s^{\infty}\right), \pi\left(t^{\infty}\right)\right]$, we are done, otherwise apply $T^{\left|s_{1}\right|}$ to $y$, etc.

If this process never stops, it means that $x=\pi\left(s_{1}^{\infty}\right)$. Let us show that its $T$-orbit falls in $\left[\pi\left(s^{\infty}\right), \pi\left(t^{\infty}\right)\right]$. Indeed, since $s_{1}$ is a cyclic permutation of $t_{1}$, there exists $j$ such that $\sigma^{j}\left(s_{1}^{\infty}\right)=t_{1}^{\infty} \prec t^{\infty}$. Also, since $t_{1}$ begins with 1 , we have $\pi\left(t_{1}^{\infty}\right)>\frac{1}{2}$. Hence $T^{j}\left(\pi\left(s_{1}^{\infty}\right)\right) \in$ $\left(\pi\left(s^{\infty}\right), \pi\left(t^{\infty}\right)\right)$.

Theorem 2.6. A Sturmian hole $H_{0}(\gamma)$ is supercritical for the doubling map $T$ for any irrational $\gamma \in(0,1 / 2)$.

Proof. (i) We want to show first that

$$
K_{\gamma}=\mathcal{J}\left(H_{0}(\gamma)\right) \backslash\{0\} .
$$

Note that

$$
\mathcal{J}\left(H_{0}(\gamma)\right) \backslash\{0\}=(0,1) \backslash \bigcup_{n=0}^{\infty} T^{-n} H_{0}(\gamma) .
$$

Firstly, we show that

$$
K_{\gamma} \cap \bigcup_{n=0}^{\infty} T^{-n} H_{0}(\gamma)=\varnothing .
$$

Since $K_{\gamma}$ is $T$-invariant, we have $T^{-n} K_{\gamma} \supset K_{\gamma}$, whence it suffices to show $T^{-n} K_{\gamma} \cap T^{-n} H_{0}(\gamma)=\varnothing$ for all $n \geq 0$. This follows from $H_{0}(\gamma) \cap K_{\gamma}=\varnothing$, which proves (2.2).

Let within this proof $s_{n}(\gamma)$ denote the standard word corresponding to $\left[d_{1}+1, \ldots, d_{n}\right]$. (Thus, $s_{n}(\gamma) \rightarrow s_{\infty}(\gamma)$ as $n \rightarrow \infty$.) Since $H_{0}(\gamma)$ is the limit of $\left(\pi\left(01\left(s_{n}(\gamma)\right)^{\infty}\right), \pi\left(10\left(s_{n}(\gamma)\right)^{\infty}\right)\right)$, and the these intervals approach $H_{0}(\gamma)$ from both ends, it follows from Lemma 2.5 that $\overline{H_{0}(\gamma)}$ is a trap. Hence $(0,1) \backslash \bigcup_{k \geq 0} T^{-k}\left(H_{0}(\gamma)\right)$ is a closure of the orbit of its endpoints, i.e., $K_{\gamma}$. This proves (2.1).

Now suppose $\overline{H_{0}(\gamma)} \subset H$; we have $\mathcal{J}\left(H_{0}(\gamma)\right) \supset \mathcal{J}(H)$, whence by (2.1), $\mathcal{J}(H) \backslash\{0\} \subset K_{\gamma}$. If $x \neq 0$ and $x \in \mathcal{J}(H)$, then $x \in K_{\gamma}$, and by the minimality of $\left.T\right|_{K_{\gamma}}$ and the fact that $\min H_{0}(\gamma) \in K_{\gamma}$, there 
exists $n \in \mathbb{N}$ such that $T^{n}(x) \in\left(\min H\right.$, min $\left.H_{0}(\gamma)\right)$, which contradicts $x \in \mathcal{J}(H)$. Hence $\mathcal{J}(H)=\{0\}$.

(ii) We define $\widetilde{X}_{\gamma, n}$ to be the set of all sequences constructed out of the blocks $s_{n}:=s_{n}(\gamma)$ and $s_{n+1}:=s_{n+1}(\gamma)$ and put $X_{\gamma, n}=\overline{\left\{\sigma^{k} w \mid k \geq 0, w \in \widetilde{X}_{\gamma, n}\right\}}$. Thus, each sequence in $X_{\gamma, n}$ is of the form $w^{\prime} w$, where $w^{\prime}=\sigma^{k} s_{n}$ or $\sigma^{k} s_{n+1}$ and $w$ is a block sequence whose each block is either $s_{n}$ or $s_{n+1}$.

We claim that

$$
X_{\gamma, n} \rightarrow X_{\gamma}, \quad n \rightarrow \infty, \quad \text { in the Hausdorff metric. }
$$

Since $\left.\sigma\right|_{X_{\gamma}}$ is minimal, for each sequence $u \in X_{\gamma}$ and each $\delta>0$ there exists $k \geq 0$ such that $\operatorname{dist}\left(u, \sigma^{k} s_{\infty}(\gamma)\right)<\delta$, where, as usual, $\operatorname{dist}(x, y)=2^{-\min \left\{j \geq 1 \mid x_{j} \neq y_{j}\right\}}$. To prove (2.3) , we first show that for each $k \geq 0$ there exists $w \in X_{\gamma, n}$ such that

$$
\operatorname{dist}\left(w, \sigma^{k} s_{\infty}(\gamma)\right)<\delta
$$

Suppose $k<q_{n}$. Then the sequence $w=\sigma^{k}\left(s_{n}\right)\left(s_{n}\right)^{\infty}$ satisfies (2.4) with $\delta=2^{-q_{n-1}}$. Indeed, $s_{\infty}(\gamma)$ always begins with $s_{n} s_{n-1} \ldots$ : if $d_{n+1}=$ 1 , then $s_{n+1}=s_{n} s_{n-1}$ and if $d_{n+1} \geq 2$, then $s_{n+1}=s_{n} s_{n} *$, and $s_{n}$ always begins with $s_{n-1}$. Hence $w$ and $\sigma^{k} s_{\infty}(\gamma)$ agree at least on the first $\left|s_{n-1}\right|=q_{n-1}$ coordinates.

On the other hand, if $w \in X_{\gamma, n}$, then $w$ begins with either $\left(\sigma^{k} s_{n}\right) s_{n-1}$ with $k<q_{n}$ or $\left(\sigma^{k} s_{n+1}\right) s_{n}$ with $k<q_{n+1}$. Since both $s_{n} s_{n-1}$ and $s_{n+1} s_{n}$ are prefixes of $s_{\infty}(\gamma)$, we conclude that in either case $\sigma^{k}\left(s_{\infty}(\gamma)\right)$ is at a distance less than or equal to $2^{-q_{n-1}}$ from $w$ again.

Now suppose $\bar{H} \subset H_{0}(\gamma)$. By (2.3), there exists $n$ such that $\pi\left(X_{\gamma, n}\right) \subset$ $\mathcal{J}(H)$. Hence $\operatorname{dim}_{H}(\mathcal{J}(H))>0$.

Remark 2.7. Note that as $H_{0}(\gamma) \rightarrow(1 / 4,1 / 2)$ from Proposition 1.5 as $\gamma \rightarrow 0$. This follows from the fact that $s_{\infty}(\gamma)$ begins with $0^{d_{1}}$ and consequently, tends to the zero sequence as $\gamma \downarrow 0$. Since a characteristic word can begin with $(01)^{n}$ for arbitrarily large $n$, we have $\sup _{\gamma} H_{0}(\gamma)=$ 1/3. Therefore,

$$
\mathcal{K}:=\left\{\min H_{0}(\gamma) \mid \gamma \in(0,1 / 2) \backslash \mathbb{Q}\right\} \subset(1 / 4,1 / 3) .
$$

Since the number of balanced words grows polynomially (see, e.g., [9, Corollary 18]), it is easy to see that $\mathcal{K}$ is a nowhere dense set of zero Hausdorff dimension.

\section{A COMPlete Description OF SUPERCRiticAl HOlES FOR THE} DOUBLING MAP

Put

$$
\mathcal{S}=\{a \in[0,1) \mid \exists b>a \text { such that }(a, b) \text { is supercritical for } T\} .
$$


It follows from Proposition 1.5 that $[0,1 / 4] \subset \mathcal{S}$ and $1 / 2 \in \mathcal{S}$. It is also clear that if $a>1 / 2$, then $a \notin \mathcal{S}$, since $\mathcal{J}(a, b)$ is of positive dimension for any $b>a$ - see the proof of Proposition 1.5 (ii).

Furthermore, since $(1 / 3,2 / 3)$ is first-order critical for $T$, it is obvious that if $a \in \mathcal{S}$ and $a \geq 1 / 3$, then the corresponding $b$ should be greater than $2 / 3$, i.e., $a+b>1$. Again, by symmetry, $(1-b, 1-a)$ is supercritical, whence it suffices to confine our search for the elements of $\mathcal{S}$ to the interval $(1 / 4,1 / 3]$.

Our goal is to prove that

$$
\mathcal{S} \cap[1 / 4,1 / 3]=\overline{\mathcal{K}}
$$

where $\mathcal{K}$ is given by (2.5).

Let $S W\left(a_{1}, \ldots, a_{n}\right)$ denote the set of all standard words of length $n$ given by some irrational $\gamma$ whose continued fraction expansion begins with $\left[a_{1}+1, \ldots, a_{n}\right]$. Thus, $s_{-1}=1, s_{0}=0, s_{k+1}=s_{k}^{a_{k+1}} s_{k-1}$ for all $k \in\{0,1, \ldots, n-1\}$. Since for $a_{n}>1$, we have $\left[a_{1}, \ldots, a_{n-1}, a_{n}\right]=$ $\left[a_{1}, \ldots, a_{n-1}, a_{n}-1,1\right]$, we assume $a_{n} \geq 2$.

Using this notation, we obtain

$$
(1 / 4,1 / 3) \backslash \mathcal{K}=\bigcup_{p / q<1 / 2,(p, q)=1}\left[\alpha_{p / q}, \beta_{p / q}\right],
$$

where $\left[\alpha_{p / q}, \beta_{p / q}\right]$ is the gap between $\left\{\pi(01 w): w \in S W\left(a_{1}, \ldots, a_{n-1}, a_{n}\right)\right\}$ and $\left\{\pi(01 w): w \in S W\left(a_{1}, \ldots, a_{n-1}, a_{n}-1\right)\right\}$.

Example 3.1. Let $p / q=1 / 3$, which implies $n=1$ and $a_{1}=2$. Denote $s_{1}=001, s_{1}^{\prime}=01$.

Then $s_{2}=(01)^{d_{2}} 0$, and $s_{3}=\left((01)^{d_{2}} 0\right)^{d_{3}} 01$, which is always lexicographically larger than $(010)^{\infty}$ (corresponding to $d_{2}=1, d_{3}=\infty$ ). Similarly, $s_{2}^{\prime}=(001)^{d_{2}} 0$, and the supremum corresponds to $d_{2}=\infty$, which yields $(001)^{\infty}$. Therefore,

$$
\left[\alpha_{1 / 3}, \beta_{1 / 3}\right]=\left[\pi\left(01(001)^{\infty}\right), \pi\left(01(010)^{\infty}\right)\right]=\left[\frac{2}{7}, \frac{9}{28}\right] .
$$

Note that $01(001)^{\infty}=(010)^{\infty}$, i.e., purely periodic. Besides, $\alpha_{1 / 3}$ and $\beta_{1 / 3}$ are points in the same periodic orbit. We will see later that this is always the case for $\alpha_{p / q}$.

Example 3.2. Let now $n=2$ and $p / q=2 / 5=[2,2]$. Here $s_{1}=$ $01, s_{2}=01010, s_{2}^{\prime}=010$. We observe the role-reversal: it is now $[2,1]$ that yields $\alpha_{2 / 5}$ and $[2,2]$ yields $\beta_{2 / 5}$. More precisely,

$$
\begin{aligned}
& \sup \left\{s_{N}: a_{1}=1, a_{2}=1\right\}=(01001)^{\infty}, \\
& \inf \left\{s_{N}: a_{1}=1, a_{2}=2\right\}=(01010)^{\infty},
\end{aligned}
$$


whence, in view of $01(01001)^{\infty}=(01010)^{\infty}$,

$$
\left[\alpha_{2 / 5}, \beta_{2 / 5}\right]=\left[\pi\left((01010)^{\infty}\right), \pi\left(01(01010)^{\infty}\right)\right]=\left[\frac{10}{31}, \frac{41}{124}\right] .
$$

Before we tackle the general case, we need the following auxiliary claim:

Lemma 3.3. Let $s_{n-1}, s_{n}$ be standard words with $s_{n}=s_{n-1}^{a_{n}} s_{n-2}$. Then

$$
\begin{array}{ll}
s_{n-1} s_{n} \prec s_{n} s_{n-1}, & n \text { odd, } \\
s_{n} s_{n-1} \prec s_{n-1} s_{n}, & n \text { even. }
\end{array}
$$

Proof. We prove both claims simultaneously by induction. It is obvious that $s_{0} s_{-1}=01 \prec 10=s_{-1} s_{0}$ and $s_{0} s_{1}=0^{d_{1}+1} 1 \prec 0^{d_{1}} 10=s_{1} s_{0}$. Assume the claim holds for all $k<n$. We have

$$
s_{n-1} s_{n}=s_{n-1}^{d_{n}+1} s_{n-2}=s_{n-1}^{d_{n}} s_{n-1} s_{n-2} .
$$

If $s_{n-1} s_{n-2} \prec($ resp. $\succ) s_{n-2} s_{n-1}$, this implies $s_{n-1} s_{n} \prec$ (resp. $\succ$ )$s_{n} s_{n-1}$.

The following lemma gives an explicit formula for $\left[\alpha_{p / q}, \beta_{p / q}\right]$ for a general $p / q$.

Lemma 3.4. We have

$$
\left[\alpha_{p / q}, \beta_{p / q}\right]=\left\{\begin{array}{lll}
{\left[\pi\left(01\left(s_{n}\right)^{\infty}\right), \pi\left(01\left(s_{n-1}^{a_{n}-1} s_{n-2} s_{n-1}\right)^{\infty}\right)\right],} & n & \text { odd }, \\
{\left[\pi\left(01\left(s_{n-1}^{a_{n}-1} s_{n-2} s_{n-1}\right)^{\infty}\right), \pi\left(01\left(s_{n}\right)^{\infty}\right)\right],} & n & \text { even } .
\end{array}\right.
$$

Proof. We show first that

$$
\sup \left\{S W\left(d_{1}, d_{2}, \ldots\right) \mid d_{1}=a_{1}, \ldots d_{n}=a_{n}\right\}=\left(s_{n}\right)^{\infty}, \quad n \text { odd },
$$

where supremum is understood in the sense of lexicographic order. We have $s_{n+1}=s_{n}^{d_{n+1}} s_{n-1}$, and $s_{n+2}=s_{n+1}^{d_{n+2}} s_{n}=s_{n}^{d_{n+1}} s_{n-1} s_{n}^{d_{n+1}} \ldots \prec$ $\left(s_{n}\right)^{\infty}$, in view of Lemma 3.3. (Since $s_{n}$ begins with $s_{n-1}$, we have $\left.s_{n-1} s_{n} \cdots \prec s_{n} s_{n} \ldots\right)$. This proves (3.2).

Similarly,

$$
\sup \left\{S W\left(d_{1}, d_{2}, \ldots\right) \mid d_{1}=a_{1}, \ldots d_{n}=a_{n}\right\}=\left(s_{n} s_{n-1}\right)^{\infty}, \quad n \text { even. }
$$

Indeed, by Lemma 3.3 , we have $s_{n}^{2} \prec s_{n-1} s_{n}$. Hence $s_{n}^{d_{n+1}} s_{n-1} s_{n}^{d_{n+1}} \ldots \prec$ $\left(s_{n} s_{n-1}\right)^{\infty}$, which proves (3.3) . Consequently,

$$
\begin{aligned}
& \sup \left\{S W\left(d_{1}, d_{2}, \ldots\right) \mid d_{1}=a_{1}, \ldots, d_{n-1}=a_{n-1}, d_{n}=a_{n}-1\right\} \\
& \quad=\left(s_{n-1}^{a_{n}-1} s_{n-2} s_{n-1}\right)^{\infty}, \quad n \text { even. }
\end{aligned}
$$

The two remaining inequalities are proved in exactly the same way. 
By induction, $s_{n}$ always ends with 01 if $n \geq 1$ is odd and with 10 otherwise. Hence $\alpha_{p / q}$ always has a purely periodic binary expansion of period $q$. Put $\gamma_{p / q}=\beta_{p / q}+1 / 4$. Clearly, the binary expansion of $\gamma_{p / q}$ can be obtained from that of $\beta_{p / q}$ by replacing the first two digits 0,1 with 1,0 . Hence by the previous lemma, the binary expansion of $\gamma_{p / q}$ is purely periodic of period $q$ as well. Thus, we have

$$
T^{q}\left(\alpha_{p / q}\right)=\alpha_{p / q}, \quad T^{q}\left(\beta_{p / q}\right)=T^{q}\left(\gamma_{p / q}\right)=\gamma_{p / q} .
$$

Put

$$
\mathcal{O}(p / q)=\left\{T^{n}\left(\alpha_{p / q}\right) \mid n \in \mathbb{N}\right\} .
$$

Lemma 3.5. We have $\mathcal{O}(p / q) \cap\left(\alpha_{p / q}, \gamma_{p / q}\right)=\varnothing$.

Proof. Since $\gamma_{p / q}$ is purely periodic, and the binary expansion of the periodic part has the length $q$ and the same number of 1 s as that of $\alpha_{p / q}$ (i.e., $p$ ), we conclude that $\gamma_{p / q} \in \mathcal{O}(p / q)$, in view of the fact that any two balanced words with the same length and the same number of 1 s are cyclic permutations of each other - see [8, Chapter 2].

Assume first $n$ to be odd; we have

$$
\alpha_{p / q}=\pi\left(01\left(s_{n}\right)^{\infty}\right)=\pi\left(\left(w_{1} \ldots w_{q}\right)^{\infty}\right) .
$$

It is well known that if $w=w_{1} \ldots w_{q}$ is standard and ends with 01, then its cyclic permutation $w_{q} w_{1} \ldots w_{q-1}$ is lexicographically largest among all cyclic permutations of $w$. Consequently, $w_{q-1} w_{q} w_{1} \ldots w_{q-2}$ is the largest cyclic permutation beginning with 0 .

The word $s_{n-1}^{a_{n}-1} s_{n-2} s_{n-1}$ has the same length and the same number of $1 \mathrm{~s}$ as $s_{n}$ (and is balanced, since it is standard), whence it is also a cyclic permutation of $s_{n}$. By the same argument, adding the prefix 10 to $w_{1}^{\prime} \ldots w_{q}^{\prime}:=s_{n-1}^{a_{n}-1} s_{n-2} s_{n-1}$ and removing its last two symbols yields the lexicographically smallest cyclic permutation $10 w_{1}^{\prime} \ldots w_{q-2}^{\prime}$ of $s_{n}$ beginning with 1.

Thus, there is no cyclic permutation which is lexicographically greater than $01 w_{1} \ldots w_{q-2}$ and less than $10 w_{1}^{\prime} \ldots w_{q-2}^{\prime}$. This implies that there is no element of $\mathcal{O}(p / q)$ between $\alpha_{p / q}$ and $\gamma_{p / q}$.

The case of even $n$ is analogous.

Corollary 3.6. For each $p / q$ we have

$$
\mathcal{J}\left(\alpha_{p / q}, \gamma_{p / q}\right)=\mathcal{O}(p / q) \cup \bigcup_{k \geq 0} T^{-k}\left(\left\{\alpha_{p / q}, \gamma_{p / q}\right\}\right) .
$$

Proof. The claim follows from Lemma 3.5 together with Lemma 2.5. 


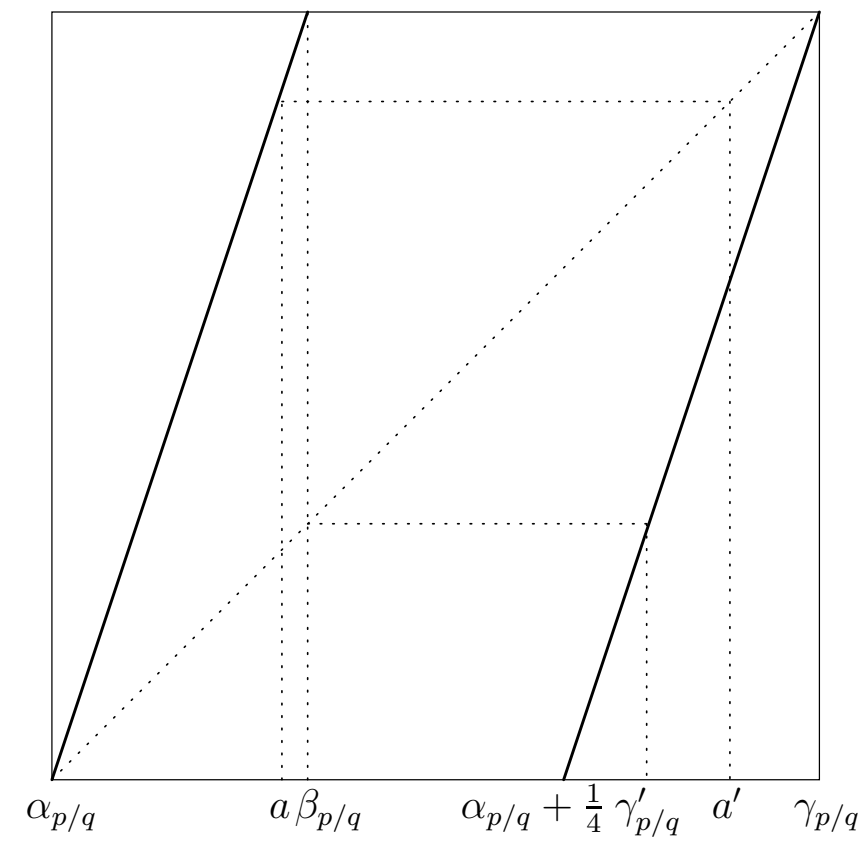

Figure 1. Part of the map $T^{q}$ restricted to the interval $\left[\alpha_{p / q}, \gamma_{p / q}\right]$

Proposition 3.7. For any $p / q$ we have

$$
\left(\alpha_{p / q}, \beta_{p / q}\right) \cap \mathcal{S}=\varnothing .
$$

Proof. Let $a \in\left(\alpha_{p / q}, \beta_{p / q}\right)$ and assume, on the contrary, that there exists $b$ such that $(a, b)$ is supercritical. Note first that $b$ cannot be less than $\gamma_{p / q}$, otherwise Lemma 3.5 implies that there exists $\delta>0$ such that $\mathcal{O}(p / q) \subset \mathcal{J}(a-\delta, b+\delta)$. Thus, $b \geq \gamma_{p / q}$.

We claim that the condition of Definition 1.4 (ii) fails here. Namely, since $T^{q}\left(\alpha_{p / q}\right)=\alpha_{p / q}, T^{q}\left(\beta_{p / q}\right)=\gamma_{p / q}$, we have that there exists a unique $k \geq 1$ such that $a^{\prime}:=T^{k q}(a) \in\left(\beta_{p / q}, \gamma_{p / q}\right)$. Let $\gamma^{\prime}(p / q)$ be such that $T^{q}\left(\gamma_{p / q}^{\prime}\right)=\beta_{p / q}$.

Then

$$
\mathcal{J}(a, b)=\mathcal{O}(p / q) \quad \forall b \in\left(\max \left\{a^{\prime}, \gamma^{\prime}(p / q)\right\}, \gamma_{p / q}\right]
$$

Indeed, by Corollary 3.6, it suffices to check that for any $x \in\left(\alpha_{p / q}, a\right) \cup$ $\left(\max \left\{a^{\prime}, \gamma^{\prime}(p / q)\right\}, \gamma_{p / q}\right)$ there exists $s \in \mathbb{N}$ such that $T^{s}(x) \in(a, b)$. If 
$x \in\left(\alpha_{p / q}, a\right)$, then we put $s=k q$; if $x \in\left(\max \left\{a^{\prime}, \gamma^{\prime}(p / q)\right\}, \gamma_{p / q}\right)$, then we put $s=q-$ see Figure 12 This proves (3.4).

Thus, our assumption was wrong, which proves the proposition.

The only points to study which remain are the $\alpha_{p / q}, \beta_{p / q}$ and $1 / 3$. We can use the following simple claim:

Lemma 3.8. Suppose $a_{j} \rightarrow a, b_{j} \rightarrow b$ as $j \rightarrow+\infty$, and that each hole $\left(a_{j}, b_{j}\right)$ is supercritical for $T$. Then $(a, b)$ is supercritical as well.

Proof. Follows directly from Definition 1.4.

Now, we know that both $\alpha_{p / q}$ and $\beta_{p / q}$ are limit points of the set $\mathcal{K}$, which implies $\left(\alpha_{p / q}, \alpha_{p / q}+1 / 4\right)$ and $\left(\beta_{p / q}, \beta_{p / q}+1 / 4\right)$ are supercritical. Consequently, $\alpha_{p / q} \in \mathcal{S}$ and $\beta_{p / q} \in \mathcal{S}$ for all $p / q$.

Example 3.9. It follows from Examples 3.1 and 3.2 that the holes $\left(\frac{2}{7}, \frac{15}{28}\right),\left(\frac{9}{28}, \frac{4}{7}\right),\left(\frac{10}{31}, \frac{71}{124}\right)$ and $\left(\frac{41}{124}, \frac{18}{31}\right)$ are all supercritical.

Finally, $1 / 3=\pi\left((01)^{\infty}\right)$, which is $\lim _{N \rightarrow \infty} \pi\left(01 s_{\infty}\left(\gamma_{N}\right)\right)$, where $\gamma_{N}=$ $[2, N, 1,1,1, \ldots]$.

Therefore,

$$
\mathcal{S} \cap[1 / 4,1 / 3]=\{1 / 4\} \cup \mathcal{K} \cup \bigcup_{p / q<1 / 2,(p, q)=1}\left\{\alpha_{p / q}, \beta_{p / q}\right\} \cup\{1 / 3\} .
$$

Note that

$$
\overline{\mathcal{K}}=\{1 / 4\} \cup \mathcal{K} \cup \bigcup_{p / q<1 / 2,(p, q)=1}\left\{\alpha_{p / q}, \beta_{p / q}\right\},
$$

which yields (3.1).

It follows from Proposition 1.5 that $(1 / 2, b)$ is supercritical for $T$ for any $b \in[3 / 4,1]$. The following proposition asserts that if $a \in \mathcal{S} \backslash\{1 / 2\}$, then the right endpoint of the corresponding supercritical hole cannot "float":

Proposition 3.10. If $a \in \mathcal{S} \backslash\{[0,1 / 4] \cup\{1 / 2\}\}$ and $(a, b)$ is supercritical for $T$, then $b=a+1 / 4$. If $a \in[0,1 / 4]$ and $(a, b)$ is supercritical for $T$, then $b=1 / 2$.

Proof. We have several cases. Within this proof we always assume that $(a, b)$ is supercritical for $T$.

(a) Let first $a \in[0,1 / 4]$. If $b<1 / 2$, then we know $\mathcal{J}(a+\delta, b-\delta)$ has a positive dimension for all $\delta<1 / 2-b$, which means $(a, b)$ cannot be supercritical.

\footnotetext{
${ }^{2}$ There may be pieces of the map $T^{q}$ between $\beta_{p / q}$ and $\alpha_{p / q}+1 / 4$ which lie in the square in question but they do not affect our argument, so we ignore them.
} 
Assume $b>1 / 2$. If $a<1 / 4$, then $\mathcal{J}(a+\delta, b-\delta)=\{0\}$ for all $\delta<\min \{a-1 / 4,1 / 2-b\}$. If $a=1 / 4$, then $T(1 / 4+\delta)=1 / 2+2 \delta \in$ $(1 / 4+\delta, b-\delta)$ for any $\delta<(b-1 / 2) / 3$.

(b) Now let $a=\alpha_{p / q}$ for some $p / q$ and assume first that $b>a+1 / 4$. Consider the hole $(a+\delta, b-\delta)$ with $b-\delta>a+1 / 4$. Since $(a, a+1 / 4)$ is supercritical, $\mathcal{J}(a-\varepsilon, a+1 / 4+\varepsilon)=\{0\}$ for any $\varepsilon>0$, whence $\mathcal{J}(a+\delta, b-\delta)=\{0\}$, because if $\delta$ is small enough, then for any $x \in(a, a+\delta)$ there exists $j \geq 1$ such that $T^{j} x \in(a+\delta, b-\delta)$. This contradicts $(a, b)$ being supercritical.

Now assume $b<a+1 / 4$. Since $(a, a+1 / 4)$ is supercritical, $\mathcal{J}(a+$ $\delta, a+1 / 4-\delta)$ is of positive dimension, therefore, non-empty. However, no $x \in(a, a+\delta)$ is in $\mathcal{J}(a+\delta, a+1 / 4-\delta)$, which means that there exists $y \in(b, a+1 / 4)$ such that the $T$-orbit of $y$ has an empty intersection with $(a+\delta, a+1 / 4-\delta)$ and consequently, with $(a+\delta, b-\delta)$. Hence $(a, b)$ cannot be supercritical. The case $a=\beta_{p / q}$ is similar.

(c) If $a=1 / 3$ and $b \neq 7 / 12$, then the argument goes exactly like case (b), and we leave it to the reader as an exercise.

(d) Finally, let $a \in \mathcal{K}$ be given by $\gamma=\left[a_{1}+1, a_{2}, \ldots\right]$, and, as usual, $s_{n}=s_{n-1}^{a_{n}} s_{n-2}, n \geq 1$. Note first that $\left(s_{n}\right)^{\infty} \prec s_{\infty}(\gamma)$ if $n$ is even and $\left(s_{n}\right)^{\infty} \succ s_{\infty}(\gamma)$ otherwise. Indeed, $s_{\infty}(\gamma)$ begins with $s_{n+1} s_{n}=$ $s_{n}^{d^{n+1}} s_{n-1} s_{n}$, and $s_{n}$ begins with $s_{n-1}$, so the inequalities in question follow from Lemma 3.3. Hence by Lemma 3.4, $a_{n}:=\alpha_{p_{2 n-1} / q_{2 n-1}} \downarrow a$ and $a_{n}^{\prime}:=\alpha_{p_{2 n} / q_{2 n}} \uparrow a$ as $n \rightarrow \infty$.

Assume now $b>a+1 / 4$; then there exists $n$ such that $a_{n}+1 / 4<b$, while $\left(a_{n}, a_{n}+1 / 4\right)$ is, as we know, supercritical. Since $\left[a_{n}, a_{n}+1 / 4\right] \subset$ $(a, b)$, this contradicts Definition 1.4. Similarly, if $b<a+1 / 4$, then $[a, b] \subset\left(a_{n}^{\prime}, a_{n}^{\prime}+1 / 4\right)$ for some $n$, which is equally impossible if $(a, b)$ is supercritical.

Remark 3.11. The case $a=1 / 3$ is rather curious. We know that the 2-cycle arises at $b_{0}=2 / 3$ and that there are no other cycles in $\mathcal{J}(a, b)$ for $b \in\left(b_{1}, b_{0}\right)$, where $b_{1}=7 / 12$ (see [1]). Once $b<b_{1}$, we immediately get from Proposition 3.10 that $\operatorname{dim}_{H} \mathcal{J}(a, b)>0$, which implies that $\mathcal{J}(a, b)$ contains infinitely many cycles. As we see, this is different from the symmetrical case.

As a corollary, we obtain a complete description of the set of all possible left endpoints of supercritical points:

Corollary 3.12. We have

$$
\mathcal{S}=[0,1 / 4) \cup \overline{\mathcal{K}} \cup(3 / 4-\overline{\mathcal{K}}),
$$


i.e., $\mathcal{S}$ is a disjoint union of an interval and a Cantor set of zero Hausdorff dimension.

Proof. It follows from Proposition 3.10 that if $(a, b)$ is supercritical for $T$, then no $\left(a^{\prime}, b\right)$ is such for $a^{\prime} \neq a$ unless $b=1 / 2$.

So, we have obtained a complete list of all supercritical holes for the doubling map:

Theorem 3.13. Each supercritical hole for the doubling map is one of the following:

(i) $(\alpha, 1 / 2)$ or $(1 / 2,1-\alpha)$ for any $\alpha \in[0,1 / 4]$;

(ii) $(\alpha, \alpha+1 / 4)$ or $(3 / 4-\alpha, 1-\alpha)$, where $\alpha$ is one of the following:

- $1 / 3$;

- $\pi\left(01 s_{\infty}(\gamma)\right)$, where $s_{\infty}(\gamma)$ is the characteristic word for an arbitrary irrational $\gamma<1 / 2$;

- $\pi\left(01\left(s_{n}\right)^{\infty}\right)$ or $\pi\left(01\left(s_{n-1}^{a_{n}-1} s_{n-2} s_{n-1}\right)^{\infty}\right)$, where $\left(s_{k}\right)_{k=-1}^{n}$ is the sequence of standard words parametrized by an arbitrary $n$-tuple $\left(a_{1}, \ldots, a_{n}\right) \in \mathbb{N}^{n}$ with $a_{n} \geq 2$. The binary expansion of $\alpha$ is an eventually periodic sequence of period $q$, where $p / q=\left[a_{1}+1, \ldots, a_{n}\right]$.

Remark 3.14. It would be interesting to find a complete list of supercritical holes for an arbitrary $\beta$-transformation $T_{\beta}(x)=\beta x \bmod 1$ with $\beta>1$. Note that some of the results of our paper can be easily transferred to such a map if $\beta<2$; for instance, similarly to Proposition 1.5, one can easily show that $H_{\beta}:=\left(\beta^{-2}, \beta^{-1}\right)$ is supercritical for $T_{\beta}$. As a corollary, we infer that a supercritical hole can have a measure arbitrarily close to 0 , since $\left|H_{\beta}\right| \rightarrow 0$ as $\beta \downarrow 1$. However, the fact that not all 0-1 sequences are admissible (see, e.g., [11]) makes this set-up significantly more complicated as far as a complete description is concerned.

Acknowledgement. The author is grateful to Henk Bruin, Paul Glendinning, Kevin Hare and Oliver Jenkinson for stimulating discussions.

\section{REFERENCES}

[1] J.-P. Allouche, M. Clarke and N. Sidorov, Periodic unique beta-expansions: the Sharkovskiu ordering, Ergod. Th. Dynam. Systems, 29 (2009), 1055-1074.

[2] S. Bullett and P. Sentenac, Ordered orbits of the shift, square roots, and the devil's staircase, Math. Proc. Camb. Phil. Soc., 115 (1994), 451-481.

[3] S. Bundfuss, T. Krueger and S. Troubetzkoy, Topological and symbolic dynamics for hyperbolic systems with holes, Ergod. Th. Dynam. Systems, 31 (2011) $1305-1323$. 
[4] P. Glendinning and N. Sidorov, Unique representations of real numbers in non-integer bases, Math. Res. Lett., 8 (2001), 535-543.

[5] K. G. Hare and N. Sidorov, On cycles for the doubling map which are disjoint from an interval, http://arxiv.org/abs/1308.2905.

[6] A. Katok and B. Hasselblatt, Introduction to the Modern Theory of Dynamical Systems, Encyclopedia of Mathematics and its Applications, 54, Cambridge Univ. Press, Cambridge, 1995.

[7] R. Labarca and C. G. Moreira, Essential dynamics for Lorenz maps on the real line and the lexicographic world, Ann. I. H. Poincaré, 23 (2006), 683-694.

[8] M. Lothaire, Algebraic Combinatorics on Words, Encyclopedia of Mathematics and its Applications, Cambridge Univ. Press, Cambridge, 2002.

[9] F. Mignosi, On the number of factors of Sturmian words, Theoret. Comp. Sci., 82 (1991), 71-84.

[10] J. Nilsson, The fine structure of dyadically badly approximable numbers, http://arxiv.org/abs/1002.4614.

[11] W. Parry, On the $\beta$-expansions of real numbers, Acta Math. Acad. Sci. Hung., 11 (1960), 401-416.

[12] K. Zyczkowski and E. Bollt, On the entropy devil's staircase in a family of gap-tent maps, Physica D, 132 (1999), 392-410.

School of Mathematics, The University of Manchester, Oxford Road, Manchester M13 9PL, United Kingdom. E-Mail: Sidorov@Manchester.aC.uK 